case of my twins, being a complete union of the sacrum and coccyx of each child. By reference to the excellent skiagram, for which I am indebted to Dr. Bailey, it is at once seen that the lumbar and sacral vertebrae are quite separate and distinct; only the coccygeal vertebrae are joined. The light spaces in the abdomen of the second child show, according to Dr. Bailey, the existence of flatulence, and the rectum seems to end in a pouch, with, presumably, a side opening into the rectum of the more perfect child. This reading is borne out clinically, because the second child was much troubled with windy colic. The pulses are not synchronous; at least, I have never been able to demonstrate it.

The interesting question arises, What is the extent of fusion of the spinal cords? I have not been able to elicit any common reflex; the reflexes, both deep and superficial, appear to be quite independent, so one is justified in supposing that, if there is any fusion at all, it must be limited to the caudae equinae, but it is quite probable that there is no fusion at all. In the case of the other two pygopagi, to which I have already referred, the fusion was thought to be limited to the caudae equinae, and the junction was much more intimate and extreme.

The children share a common anus; they have each a separate vulva and vagina; the two labia majora of the one meet those of the other posteriorly. There is an inch of perineum between this junction of the labiae and the anus. Rosa-Josepha have one vulva, but two vaginae. Although the anus is common the rectum is double I think the act of defaecation establishes that fact, and the evidence of the skiagram is in favour of it. An inch above the anus is a well-defined dimple, which I take to be a rudimentary anus; and the same distance above that another dimple, which occasionally discharges a minute quantity of matter having an offensive smell. This discharge is only noticed when the children are out of health. A probe can be passed $\frac{1}{4}$ inch upwards and forwards.

Of all the pygopagi observed, the bond of union seems to be slightest in these Brighton twins; they approach most nearly to the normal. In the British Medical Journal of April 30th, 1910, there is an account of a very interesting case seen by Surgeon Riggall, R.N., at Hong Kong. It is a case of male pygopagi; it is not quite clear what the union comprises, whether the vertebrae are involved, but the interesting point is that they also have a common anus and a common rectum. They are otherwise apparently normal and can walk.

In conclusion I may add a short note on the family history of the mother. Tĥe grandmother of the children had four separate births of twins, as well as other children, and was herself a twin. The mother of the children was one of these twins. I am told by a member of the staff of the hospital where the father attended that he had well. marked stigmata of degeneration, but there is no history of twin births in his family. He is since dead.

At the present time the children, who are $2 \frac{1}{2}$ years old, show no signs of being able to walk, or of any desire to attempt it. The utmost they can do is to stand propped up by a wooden framework which practically supports them. They are bright and intelligent, the elder one especially, and can talk as much as any child of their age. The shape of the head is rather peculiar, the forehead bulging and the vertex flat; the children have almost a hydrocephalic appearance.

The question of the possibility of separation was raised at a meeting of the Sussex Medico-Chirurgical Society, and the unanimous opinion of all present was that it would be an unjustifiable operation, and would certainly result in the death of one child, and probably of both-an opinion with which, in view of our ignorance of the internal arrangements of the viscera and blood vessels of these children, I certainly agree.

THE personal estate of the late Sir George Plunkett O'Farrell, an obituary notice of whom appeared in our issue for July 8th, has been sworn at a value of $£ 20,205$.

SuRgeon James C. Bringan, M.B., has received the King's permission to accept the Insignia of Officer of the Order of the Crown of Italy, conferred upon him by the King of Italy in recognition of valuable services rendered by him on the occasion of the earthquake in Southern Italy in the year 1908.

\section{THE TREATMENT OF SYPHIIIS BY SALVARSAN.}

BX

CARL H. BROWNING,

LECTURER ON BACTERIOLOGY, GLASGOW UNIVERSITY, AND

IVY McKENZIE,

DIRECTOR OF THE WESTERN ASYLUMS' RESEARCH LABORATOIY, GLASGOW.

DURING the past fifteen months about 300 cases have been treated with salvarsan. These include almost all the early and late manifestations of syphilis.

\section{Primary Sore.}

The number of cases treated, in which the only clinical evidence of infection was the primary sore, is 22 . Two of these received one injection of 0.3 gram as clear alkaline solution intramuscularly, four received $0.4 \mathrm{gram}$ as neutral suspension subcutaneously, the rest were treated by intravenous injection with doses varying from 0.3 to 0.6 gram in dilute alkaline solution (0.1 gram salvarsan in $50 \mathrm{c.cm}$. normal saline solution). In none of the 22 cases has any symptom appeared since the treatment. The 2 cases treated intramuscularly have been quite well for almost a year, and the serum reaction was negative in both six months after the injection. In 3 of the 4 cases treated with neutral suspension the serum reaction was negative six months after injection. In the fourth case the reaction was still positive after six months. An intravenous injection of 0.5 gram was given, and a month later the serum reaction was negative in this case also. All of the 16 patients treated intravenously received a second injection after three or four weeks. The serum reaction in 7 out of 10 was negative a month after the second injection. The other cases have not yet been examined.

Secondary Stage.

The number of cases of early syphilis treated in which, in addition to the chancre, there were other signs of infection-namely, fever, headache, sore throat, and exanthemata-is 80 ; of these 6 received, in the first instance, intramuscular injections of clear alkaline solution; 17 received neutral suspension in the scapular region; the rest were injected intravenously. The dosage varied from 0.3 to 0.6 gram, the majority receiving 0.4 to 0.5 gram. Of the 17 cases which received neutral suspension, 8 subsequently received intravenous injections. It is impossible to classify these cases according to the method of injection or the dosage, because some patients were treated by several methods, and the doses depended on the weight and sex of the patients. The general result of treatment in these 80 cases was an almost immediate disappearance of all the symptoms after a single injection. In every instance the chancre healed within a fortnight, and in some cases in five days. Macular and roseolar eruptions disappeared within ten days. Ulcers in the mouth healed within ten days. In 3 of the cases treated by subcutaneous injection of $0.3 \mathrm{gram}$ as neutral suspension, relapse occurred before the treatment was repeated. In 1 case this occurred eight weeks after the first injection, and took the form of iridocyclitis of one eye and slight optic neuritis of the other. A second injection of 0.4 gram salvarsan as neutral suspension caused the symptoms to disappear in a week. Eight weeks later iridocyclitis reappeared, now affecting both eyes. We did not have the opportunity of seeing the patient at the time of the second recurrence. He was treated with mercury, which caused some improvement, but after six months of such treatment the serum reaction was still positive. It is important to note in connexion with this case that the drug in neutral suspension was imperfectly absorbed on both occasions. Considerable swellings were present at the sites of injection several months after treatment. The other 2 cases which relapsed had also been treated with neutral suspension, and in each case the drug had been imperfectly absorbed. Intravenous injections produced immediate disappearance of symptoms in both cases, and the patients are still well six months later. 
Late Secondary Stage.

The number of cases treated in the late secondary stage is 32. Each of these had been refractory to previous treat ment with mercury. They presented severe lesions of the skin and mucous membranes-for example, deep ulcers involving the subcutaneous tissues, and extensive necrosis of tonsils, soft palate, and turbinate bones. In every case salvarsan produced complete arrest and healing of the local condition. As regards the method of treatment, 7 received subcutaneous injections of neutral suspension in the first instance, and 6 of these received an intravenous injection subsequently. The remaining 25 were each treated by two intravenous injections. In 18 out of the 32 cases the serum reaction was negative one to three months after the second injection; in 6 cases the reaction was still positive three months after the second injection; in the remaining cases the time which has elapsed since treatment is too short to admit of any conclusion.

A woman with secondary syphilis and in the sixth month of pregnancy was treated with 0.4 gram intravenously, repeated after five weeks. A healthy child was born at full time, and six months after the birth both mother and child were in good health.

\section{Tertiary Stage.}

The number of cases treated in the tertiary stage is 14 , including gummata, indolent ulcers, periosteal thickenings, and severe forms of neuralgia. The gummata disappeared and the ulcers healed in every instance. One case of severe trigeminal neuralgia in a woman who had never had any symptoms of syphilis, but whose serum gave a positive reaction, was cured after an intramuscular injection of 0.5 gram in clear alkaline solution. Severe neuralgic pains in the legs in two syphilitics, twenty years aiter infection, disappeared after one intravenous injection of 0.4 gram in each case.

Late Nervous Disorders.

The number of cases of general paralysis of the insane treated is 58 . In 12 patients there has been a marked amelioration of symptoms, and 6 of these are now free from restraint and pursuing their ordinary work. In addition, 7 cases of locomotor ataxy have been treated. In 3 there has been marked improvement. It must be noted, however, that while distinct benefit has been obtained in "parasyphilitic" conditions, the results are much inferior to those in the more recent manifestations.

\section{Congenital Syphilis.}

Three cases of congenital syphilis with late lesions have been treated. Here also the results are less satisfactory than in recent acquired syphilis. The symptoms in such cases disappear slowly, and the serum reaction tends to remain positive. One case of an extensive skin syphilide in a boy of 15 healed excellently, and the serum reaction became negative after one subcutaneous injection ( 0.45 gram, which was poorly absorbed) and two intravenous injections (0.2 and 0.27 gram).

\section{Advantages and Risks of Salvarsan.}

The effect of the injection is, in most instances, im mediate, and intravenous administration causes practically no discomfort. One injection is more efficacious than a prolonged course of mercury. Symptoms dis appear and ulcers heal rapidly; not merely does this diminish chances of spreading infection, but it saves the patient from the grave consequences of chronic lesions Cases which have proved refractory to mercury generally yield at once to salvarsan. By intravenous injection the drug is brought into contact with the tissues in maximum concentration; hence the conditions are most favourable for sterilization.

We have given about 400 intravenous injections, and have observed no serious result. The only case showing severe symptoms was that of a man who was seized a few hours after the second injection with severe sickness and vorniting, accompanied by considerable collapse. On the following day jaundice was present. In three days the symptoms had passed off. The first injection had been well borne in this case. A complication which may follow the subcutaneous administration of neutral suspension is the occurrence of extensive necrosis of the skin and the adjacent tissue. Hence this method of injection cannot be recommended. An examination of the literature shows that in most instances fatal results occurred in cases which were in a hopeless condition prior to treatment, or in which the drug had been wrongly administered. Taking into consideration also the fact that salvarsan, as we have been informed by Messrs. Meister, Lucius, and Brüning, has been administered about a million times during the first year of its use, we consider that the unfavourable criticisms regarding the toxicity of the drug are witbout foundation. This applies to statements regarding danger both of death and also of nerve damage following treatment with salvarsan. The drug compares favourably with cocaine and antipyrin as regards safety of administra. tion, and it is probably safer than ether or chloroform.

\section{Method of Treatment.}

Much light has recently been thrown on the latency and chronicity of syphilitic infections. It is probable that mercury, especially as ordinarily administered by the mouth, in the majority of cases renders the infection merely latent, so that there is still the possibility of manifestations of the disease occurring at a later date. The object of treatment is to produce complete sterilization. We would recommend as the best possible means to this end a combined therapy. The treatment should be begun as soon as possible. Where there is the suggestion of a primary syphilitic lesion, even if spirochaetes cannot be found and the serum reaction is negative, treatment should be begun without delay. An intravenous injection of alkaline solution of salvarsan should be given ( 0.4 to 0.5 gram in the case of a man), and this should be repeated in three weeks. After each injection a course of mercurial inunction should be given. Of course, attention should be paid to general hygiene, and there should be abstention from tobacco and alcohol.

The serum test is indispensable for controlling the effect of treatment. It is not enough that the serum should be negative on one occasion; it must remain negative. As the result of inefficient treatment the serum may become negative only temporarily. An examination of the serum should be made at periods of three to six months, and again a year after all symptoms have disappeared. It cannot be too strongly emphasized that the syphilis reaction must be carried out by those experienced in the use of a reliable method; abbreviated and simplified tests are likely only to prove misleading. Thus, in our experience there is no method so delicate and so uniformly reliable as that involving the use of lecithin and cholesterin, which has been described by Dr. Cruickshank and ourselves.

\section{SALVARSAN IN LEPROSY.}

F. A. DE VERTEUIL, M.R.C.S.ENG., MEDICAL SUPERINTENDENT, LEPER ASYLUM, COCORITE, TRINIDAD, AND

\section{F. L. DE VERTEUIL, M.D.EdIN., M.R.C.S.ENG.,} L.R.C.P.LOND., SURGEON, R.N. (RET.).

ARsEnIC in its various forms is a drug that has often been employed in the treatment of leprosy, but it has had little or no influence in arresting the slow but steady progress of the malady. A few cases have, however, been recorded in which it apparently gave satisfactory results. We ourselves have tried in a few selected cases injections of sodium cacodylate and atoxyl. The beneficial results obtained in these cases made us anxious to try salvarsan (dioxy-diamido-arsenobenzol).

The opportunity was afforded by the arrival at Trinidad in December, 1910, of Dr. Rost, of the German cruiser Freya, who had a number of tubes of the preparation which had been given to him at Kiel for trial in tropical diseases. He kindly placed some of these at our disposal, came himself to the asylum, and instructed us in the technique of injection.* Wechselmann's method ${ }^{1}$ was used, the powder being ground in a sterile mortar into which about 6 to $7 \mathrm{c} . \mathrm{cm}$. of sterile oleum olivarum was gradually poured, and thoroughly rubbed under slight pressure until a fine emulsion was obtained. The needle used for the injection was of rather wide calibre. The preparation of the * During lris stay in Triaidad Dr. Rost, on the advice of one of us, also injected cases of yôws at the St. Augustine Hospital. The wonderful results obtained in these cases have been recently puhlished by Dr. Alston (BRTTISH MEDICAI JOURNAL, February 18th and March
18th, 1910). 